\title{
Reflective properties of hollow microspheres in cool roof coatings
}

\author{
Olof Sandin, Jan Nordin $\mathbb{D}$, Magnus Jonsson
}

(C) The Author(s) 2017. This article is an open access publication

\begin{abstract}
Hollow ultra-low-density thermoplastic microspheres can be used as filler in elastic waterproof coatings to improve a number of important properties of the coatings. The hollow microspheres help reflect solar radiation and reduce the temperature in cool roof coatings. This can improve the indoor climate of buildings without air conditioning, or save energy by reducing the need for air conditioning. The mechanism for the reflection is described in this paper and is compared with the mechanism for reflection by pigment particles like titanium dioxide $\left(\mathrm{TiO}_{2}\right)$. Results from solar reflection measurements show that the coating system filled with hollow microspheres reflects solar radiation of all incoming wavelengths, which is different from how $\mathrm{TiO}_{2}$ reflects light. The number of reflecting units is still important for the total solar reflection and suggests that the average size of the microspheres should be as small as possible. Like most other fillers, hollow low-density thermoplastic microspheres can be used in combination with pigments.
\end{abstract}

Keywords Cool roof coatings, Expandable microspheres, Hollow microspheres, Reflective properties

\section{Introduction}

Expancel Microspheres ${ }^{\circledR}$ are thermally expandable core/shell particles in which a blowing agent, typically

This paper was presented at the 11th Coatings Science International Conference (COSI) on June 22-26, 2015 in Noordwijk, the Netherlands.

O. Sandin, J. Nordin ( $\varangle)$, M. Jonsson

AkzoNobel Pulp and Performance Chemicals, Box 13000,

85013 Sundsvall, Sweden

e-mail: jan.nordin3@akzonobel.com a low boiling hydrocarbon such as isobutane, is encapsulated by a thermoplastic polymer shell. ${ }^{1-3}$ When heated above the glass transition temperature of the polymer shell, the internal pressure from the encapsulated blowing agent causes the microsphere to expand tremendously. As the expanded form is retained upon cooling, the density of the microspheres can be reduced from about $1100 \mathrm{~kg} / \mathrm{m}^{3}$ down to around $30 \mathrm{~kg} / \mathrm{m}^{3}$. Thermally expandable microspheres are available in different forms. Hollow preexpanded microspheres are commonly used in many coating applications, whereas in other applications in which the process involves heating sufficient to expand the microspheres, unexpanded microspheres can be used. ${ }^{4}$

A modern phenomena found in cities in the warm parts of the world is the so-called urban heat island effect, where local temperature is higher than in the surrounding rural areas. The increase in temperature is created by the heat-up of buildings, roads, and other heat-absorbing infrastructure, as well as by human activities. To get comfortable living conditions, more energy is consumed in the form of air conditioning. In fact, air conditioning accounts for up to $70 \%$ of residential energy consumption in warm climates. ${ }^{5-8}$

One way to reduce heat-up of buildings is to use cool roof coatings. These coatings are designed to reflect a significant part of the energy from the sun, often aided by the addition of a reflecting pigment like titanium dioxide $\left(\mathrm{TiO}_{2}\right)$. One of the more unexpected characteristics of hollow microspheres is that they are efficient in reflecting solar energy. As this article will show, they can actually be even more efficient in reflecting solar energy than $\mathrm{TiO}_{2}$. Furthermore, although it is a known fact that white surfaces stay cooler in the sun as compared to surfaces in dark colors, the use of hollow microspheres enables tinted cool roof coatings.

Porous ceramic materials have successfully been used as insulating material around engines and 
furnaces to protect surrounding technology from heat. Dombrovsky ${ }^{9}$ has shown that the protective power is not only explained by the insulating effect of the gas filled pores in the material, but the porous material also efficiently reflects IR radiation.

It is the intention of the present paper to show that by using micron-sized hollow microspheres as filler in coatings, it is possible to enhance the reflection of solar radiation considerably, similar to the effect described for porous ceramics.

\section{Experimental section}

\section{Methods}

The coating formulations made for solar reflection measurements were made of binder (AkzoNobel Nordsjö Tinova V purchased from a local paint shop), $\mathrm{TiO}_{2}$ (Tioxide TR 88 from Huntsman Co.) dispersion and $30 \mathrm{vol} \%$ fillers such as calcium carbonate $\left(\mathrm{CaCO}_{3}\right.$, Durcal 5 from Omya), hollow glass spheres [ 3 M S38 (average particle size approx. $40 \mu \mathrm{m}$ ), 3 M K1 (average particle size approx. $100 \mu \mathrm{m}$ )], hollow thermoplastic microspheres [Expancel ${ }^{\circledR} 461$ WE from AkzoNobel Pulp and Performance Chemicals average particle size of approx. 10 (experimental sample), 20, 40, and $80 \mu \mathrm{m}$, respectively], and ceramic spheres (Insuladd from Insuladd Environmental Products Ltd., average particle size approx. $100 \mu \mathrm{m}$ ). All components were used as received. When hollow microspheres are mentioned further on in this paper, unless otherwise noted, this refers to Expancel microspheres with an average particle size of $20 \mu \mathrm{m}$.

The $\mathrm{TiO}_{2}$ dispersion was prepared by mixing $240 \mathrm{~g}$ Tioxide TR $88,8.5 \mathrm{~g}$ orotan (acting as a dispersing agent) and $50 \mathrm{~g}$ of $\mathrm{H}_{2} \mathrm{O}$ in a Silverson SL2T mixer for $5 \mathrm{~min}$. The coating formulations were mixed using a dissolver disk operated at $1800 \mathrm{rpm}$ for $5 \mathrm{~min}$. The coating samples were then placed in a vacuum chamber to remove air from the coatings. Draw downs of the coatings were made (three per sample) on Leneta opacity charts. The wet thickness of the draw downs were made in the range $1.5-0.25 \mathrm{~mm}$. The thickness of the dried draw downs were $0.6-0.15 \mathrm{~mm}$.

Directional/hemispherical reflection measurements were conducted at the Bavarian Center for applied energy research (ZAE Bayern). The UV-Vis-NIR (300-2500, $5 \mathrm{~nm}$ resolution) reflectance measurements were also conducted at Uppsala University using a Perkin-Elmer Lambda 900 spectrophotometer equipped with an integrating sphere. IR reflectance was measured by using a Bruker Tensor 27 FTIR instrument with an integrating sphere.

\section{Results and discussion}

For a cool roof coating to be effective, it is of interest to have reflection not only of visible light, but also in the near-IR region as nearly half of the incoming solar energy that hits the surface of earth is found in this region (Fig. 1). While $\mathrm{TiO}_{2}$ is a terrific reflector of visible light, it does absorb UV light. Furthermore, as shown in Fig. 2, the $\mathrm{TiO}_{2}$ evaluated in this study does not reflect near-IR radiation very well. In fact, at higher wavelengths $\mathrm{TiO}_{2}$ and $\mathrm{CaCO}_{3}$ show similar reflective properties. $\mathrm{CaCO}_{3}$ is generally considered to be a rather poor reflecting system. The effect from $\mathrm{TiO}_{2}$ can be improved by increasing the amount in the coating from the $1.5 \mathrm{wt} \%$ used in this study. However, addition of hollow thermoplastic microspheres in a corresponding amount, which also corresponds with the amount of $\mathrm{CaCO}_{3}$ based on volume, show good reflection over the entire solar spectrum. It particularly stands out from the other additives in the near-IR region.

The particle size is important for the reflective properties of hollow microspheres. By decreasing the average particle size of the spheres, the solar reflectance is increased (Figs. 3 and 4). Smaller microspheres result in a larger number of microspheres in the coating for a given volume added. This is also true for other types of hollow particles, for instance glass microspheres as can be seen by the total solar reflectance $\left(R_{\text {sol }}\right)$ measurements presented in Fig. 4. However, there is a significant difference in density when comparing these hollow thermoplastic or glass microspheres. A glass sphere with a diameter of $40 \mu \mathrm{m}$ has a much higher density compared with thermoplastic microspheres and therefore adds more weight to the paint system.

These results are consistent with the reflection properties of foam. Hollow microspheres exert foamlike behavior regarding their reflective properties, both being a multiple scattering medium. Liquid foam consists of thin-walled bubbles which encapsulate air, very similar to the thermoplastic microspheres. The difference being that the shell of the microspheres consists of a thin copolymer film and that the encapsulated gas is a hydrocarbon. The key behind the highly

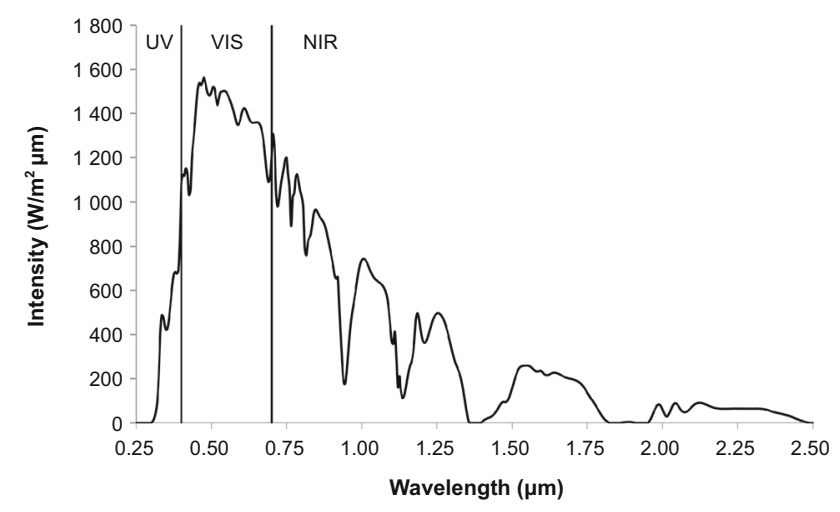

Fig. 1: Solar energy distribution. About $5 \%$ of the incoming energy is found in the UV region, $43 \%$ in the visible region, and some $52 \%$ is found in the near-IR region 


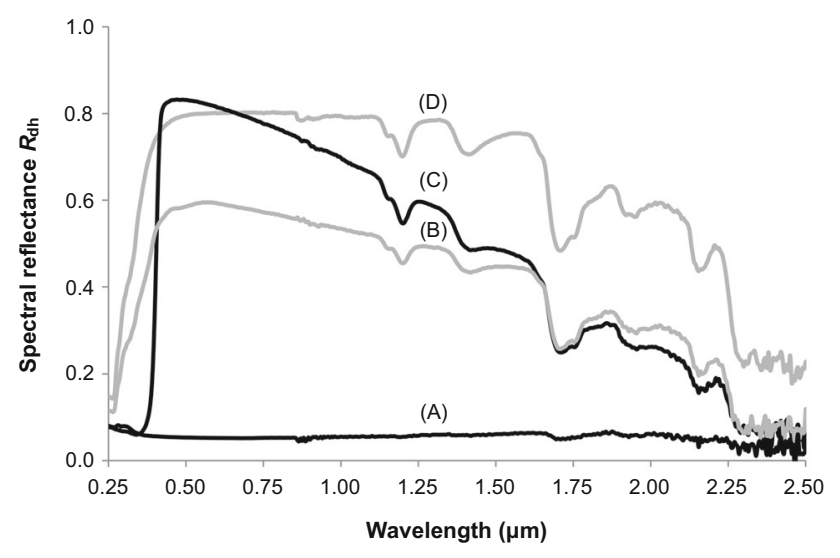

Fig. 2: Spectral reflectance measurements on coatings (dry thickness $0.8 \pm 0.05 \mathrm{~mm}$ ) prepared from (A) pure acrylic binder, (B) binder with 30 vol\% $\mathrm{CaCO}_{3}$, (C) binder with $1.5 \mathrm{wt} \% \mathrm{TiO}_{2}$, and (D) binder with 30 vol\% (approx. 1.5 wt $\%$ ) hollow microspheres

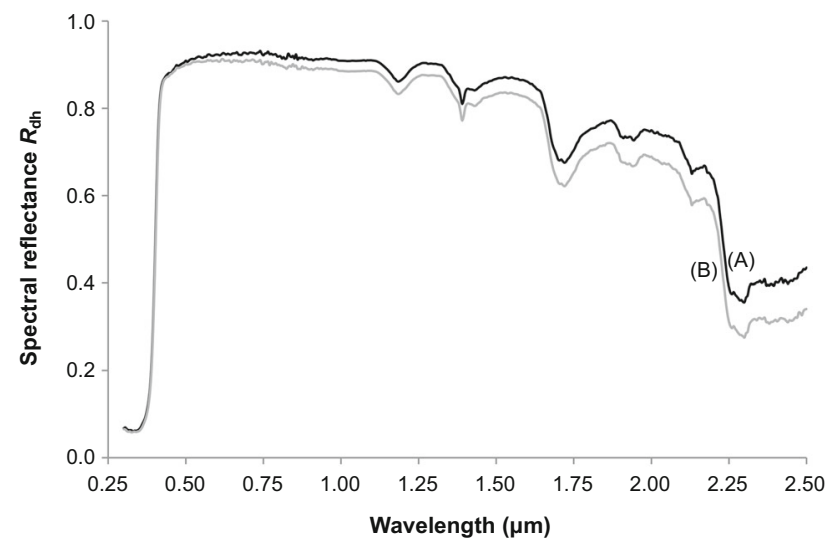

Fig. 3: Spectral reflectance in coatings (dry thickness $0.6 \mathrm{~mm} \pm 0.05 \mathrm{~mm}$ ) containing 1.6 vol\% $\mathrm{TiO}_{2}$ and $30 \mathrm{vol} \%$ hollow thermoplastic microspheres with different average particle size. (A) $20 \mu \mathrm{m}$, (B) $80 \mu \mathrm{m}$

reflective behavior of foam is the thin wall of the bubbles which makes absorption minimal. ${ }^{10}$ The difference in refractive index between the encapsulated gas, the sphere wall, and the surrounding medium refracts the light. Depending of the angle of incidence, between about $4 \%$ and $100 \%$ of the light is mirror reflected at the interface. One foam bubble has little effect, but the vast amount of bubbles present in foam clearly has an impact on the incoming light. The white color of foam illustrates this behavior.

$\mathrm{TiO}_{2}$ and other inorganic pigments reflect light differently. The diffuse reflection in a system of pigment particles is a combination of refraction and diffraction phenomena. Refraction is the bending of light traveling through a particle and is predominantly determined by the difference in refractive index of the pigment particle and the surrounding media, which is why a Rutile particle system is so efficient. Diffraction is the bending

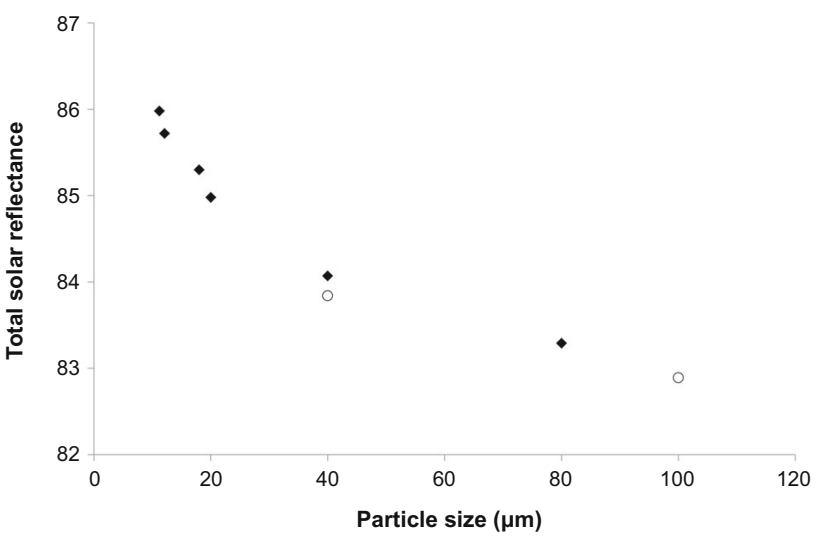

Fig. 4: Effect of particle size on the total solar reflection $\left(\boldsymbol{R}_{\text {sol }}\right)$ in coatings (dry thickness $0.6 \mathrm{~mm} \pm 0.05 \mathrm{~mm}$ ) containing $1.6 \mathrm{vol} \% \mathrm{TiO}_{2}$ and $30 \mathrm{vol} \%$ of hollow fillers having different average particle size. Paints containing smaller microspheres have higher reflectance values as these contain more reflecting units. $(\diamond)$ Hollow thermoplastic microspheres, $(\bigcirc)$ Hollow glass microspheres

of light passing near the particle and is very dependent on particle size. For the most effective light scattering, the pigment diameter should be slightly less than onehalf the wavelength of the light to be scattered. ${ }^{11}$ The light-scattering efficiency of Rutile $\mathrm{TiO}_{2}$ pigment particles is also controlled by a number of other factors, including the particle size distribution, the degree of particle agglomeration, and the degree of near-field optical interaction between neighboring particles in a pigmented microstructure. ${ }^{12}$ To optimize light scattering of visible light around $0.55 \mu \mathrm{m}$, the average particle size of commercial pigment particles for paint and coatings is in the range $0.2-0.3 \mu \mathrm{m}$. This relation implies that for a dispersion of pigment particles optimized to reflect visible light, a high-opacity system, reflection power reduces drastically for longer wavelengths. This is demonstrated in Fig. 2 where it is obvious that the reflection power for $\mathrm{TiO}_{2}$ is drastically reduced in the near-IR region. Although it is possible to improve light scattering in the near-IR region by increasing the average particle size and increasing the total addition of $\mathrm{TiO}_{2}$, this is most often not desirable as it increases the cost of the formulation. Thus, by using hollow microspheres in combination with $\mathrm{TiO}_{2}$, it is possible to formulate cost-effective cool roof coatings having excellent reflective properties. This has been demonstrated in Latin America where a producer of elastomeric cool roof coatings was able to improve the reflective properties of a coating, even though the $\mathrm{TiO}_{2}$ content was reduced from $70 \mathrm{~g} / \mathrm{L}$ down to $40 \mathrm{~g} / \mathrm{L}$ (Fig. 5).

The efficient reflection of near-IR radiation by hollow microspheres enables tinted cool roof coatings. Obviously tinted coatings will not be as effective as a white coating, since a significant part of the incoming energy in the visible region will be absorbed by the pigment. This is demonstrated for blue coatings prepared and color-matched by a local paint shop 


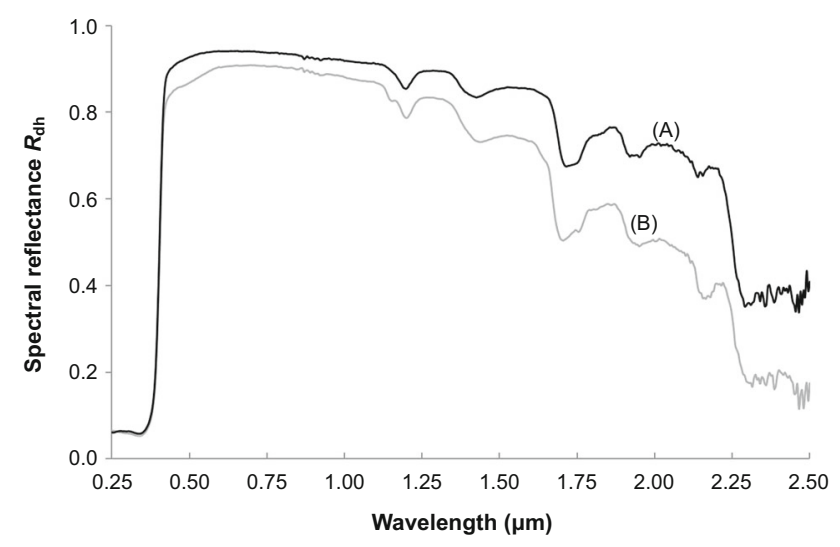

Fig. 5: Comparison of two commercial cool roof coatings from a Latin American producer of elastomeric cool roof coatings (dry thickness $0.8 \pm 0.05 \mathrm{~mm}$ ). (A) Formulation containing $40 \mathrm{~g} / \mathrm{L} \mathrm{TiO}_{2}$ in combination with 40 vol\% hollow microspheres, (B) Formulation containing $70 \mathrm{~g} / \mathrm{L} \mathrm{TiO}_{2}$ and no hollow microspheres

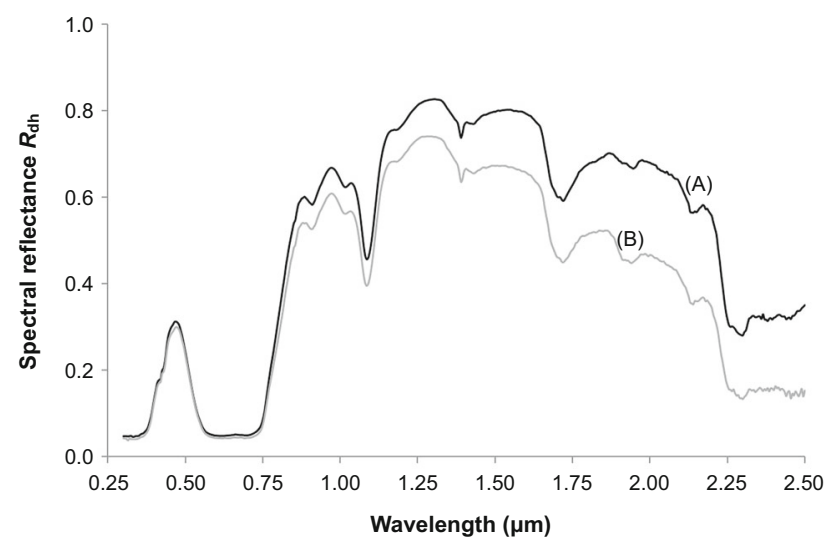

Fig. 6: Reflective properties of color-matched blue coatings (dry thickness $0.6 \pm 0.05 \mathrm{~mm}$ ) containing $30 \mathrm{vol} \%$ of (A) hollow thermoplastic microspheres, or (B) $\mathrm{CaCO}_{3}$, respectively. Measurements were performed at Uppsala University, Sweden

(Fig. 6). As expected, a significant part of the light in the visual part of the spectrum is absorbed, apart from the peak at around $450 \mathrm{~nm}$ which is responsible for their blue appearance. The similarity in the visible region indicates that the coatings are well colormatched (Fig. 6). Yet again it is shown that the hollow microspheres excel in total solar reflection when compared to other types of fillers such as $\mathrm{CaCO}_{3}$ and ceramic microspheres (Figs. 6 and 7).

Figure 7 presents the calculated total solar reflectance $\left(R_{\text {sol }}\right)$ values for color-matched blue coatings. A number of fillers have been evaluated: $\mathrm{CaCO}_{3}$, glass microspheres (average particle size $40 \mu \mathrm{m}$ ), ceramic microspheres, and thermoplastic microspheres. The relatively low $R_{\text {sol }}$ values are due to low reflectance values in the visible part of the spectrum which is taken

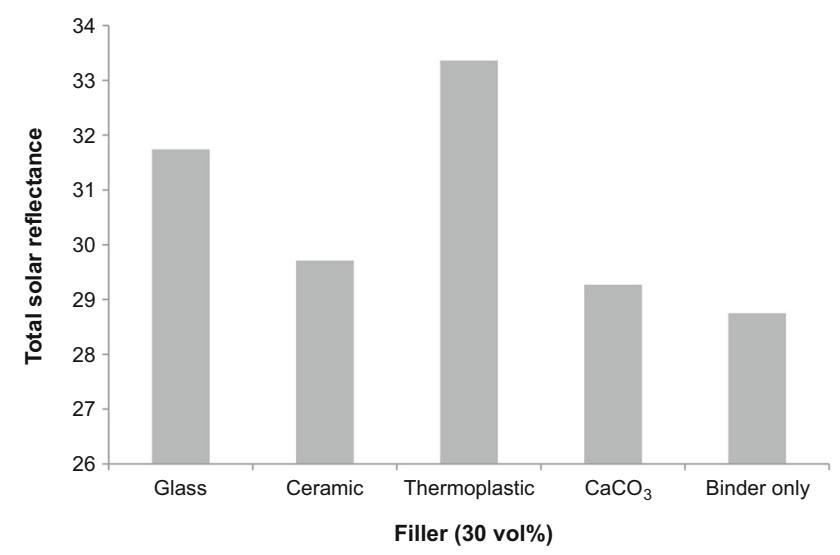

Fig. 7: Total solar reflectance $\left(\boldsymbol{R}_{\text {sol }}\right)$ calculated from the measurements of color-matched blue coatings (dry thickness $0.6 \pm 0.05 \mathrm{~mm}$ ) containing $\mathbf{3 0}$ vol\% of hollow glass microspheres, hollow ceramic microspheres, hollow thermoplastic microspheres, and $\mathrm{CaCO}_{3}$, respectively. Also included is a similarly color-matched coating prepared from the binder alone. Measurements were performed at Uppsala University, Sweden

into consideration when calculating $R_{\text {sol }}$. These coatings all absorb a great deal of the incoming visible light but the near-IR part of the spectrum is not greatly affected by the blue pigment. If the visible part was ignored when calculating $R_{\text {sol }}$, the difference between the samples in Fig. 7 would have been larger.

\section{Conclusions}

The results show that micron-sized hollow microspheres reflect solar radiation much better than dense fillers, not just in traditional white roof coatings but also in tinted coatings. The hollow microspheres reflect radiation like foam and reflect light over a wide wavelength range. In contrast to white inorganic pigments such as $\mathrm{TiO}_{2}$, the foam-like system of hollow microspheres efficiently and simultaneously reflects UV, Vis, and NIR radiation. It is obvious that the size of the microspheres is important for the reflection, where smaller bubbles make higher numbers of reflecting units per volume added and thus more efficient reflection.

Acknowledgments The authors would like to thank Annica Nilsson at Uppsala University, Solid State Physics, for letting us use their instrumentation.

Open Access This article is distributed under the terms of the Creative Commons Attribution 4.0 International License (http://creativecommons.org/ licenses/by/4.0/), which permits unrestricted use, distribution, and reproduction in any medium, provided you give appropriate credit to the original author(s) and the source, provide a link to the Creative Commons license, and indicate if changes were made. 


\section{References}

1. Morehouse Jr., DS, Midland, M, Tetreault, RJ, “Expansible Thermoplastic Polymer Particles Containing Volatile Fluid Foaming Agent and Method of Foaming the Same." US Patent 3,615,972, 1971

2. Kawaguchi, Y, Oishi, T, "Synthesis and Properties of Thermoplastic Expandable Microspheres: The Relation Between Crosslinking Density and Expandable Property." J. Appl. Polym. Sci., 93 (2) 505-512 (2004)

3. Jonsson, M, Nordin, M, Malmström, M, Hammer, C, "Suspension Polymerization of Thermally Expandable Core/Shell Particles." Polymer, 47 (10) 3315-3324 (2006)

4. https://www.akzonobel.com/expancel/ (Accessed October 17, 2016)

5. Mirzaei, PA, Haghighat, F, "Approaches to Study Urban Heat Islands-Abilities and Limitations." Build. Environ., 45 (10) 2192-2201 (2010)

6. Oke, TR, Johnson, GT, Steyn, DG, Watson, ID, "Simulations of Urban Heat Island Under 'Ideal' Conditions at Night Part 2: Diagnosis of Causation." Bound. Layer Meteorol., 56 (4) 339-358 (1991)
7. Synnefa, A, Santamouris, M, Apostolakis, K, “On the Development, Optical Properties and Thermal Performance of Cool Colored Coatings for the Urban Environment." Sol. Energy, 81 (4) 488-497 (2007)

8. Akbari, H, Pomerantz, M, Taha, H, "Cool Surfaces and Shade Trees to Reduce Energy Use and Improve Air Quality in Urban Areas." Sol. Energy, 70 (3) 295-310 (2001)

9. Dombrovsky, LA, "Approximate Models of Radiation Scattering in Hollow-Microsphere Ceramics." High Temp., 42 (5) 776-784 (2004)

10. Bohren, CF, Clouds in a Glass of Beer: Simple Experiments in Atmospheric Physics. Dover Publications Inc., Mineola (2001)

11. McNeil, LE, French, RH, "Multiple scattering from Rutile $\mathrm{TiO}_{2}$ particles." Acta Mater., 48 (18-19) 45714576 (2000)

12. Thiele, ES, French, RH, "Light-Scattering Properties of Representative, Morphological Rutile Titania Particles Studied Using a Finite-Element Method, Theory and Modeling of Glasses and Ceramics." J. Am. Ceram. Soc., 81 (3) 469-479 (1998) 